\title{
Calendar of Forthcoming Meetings Powder Diffraction September 2014
}

\author{
Gang Wang \\ Research \& Development Center for Functional Crystals, Institute of Physics, Chinese Academy of Sciences, No.8 Nansanjie, \\ Zhongguancun, Haidian District, Beijing 100190, China \\ gangwang@iphy.ac.cn
}

\section{5-10 October 2014}

10th World Conference on Neutron Radiography (WCNR-10) Grindelwald, Switzerland [Info: http://indico. psi.ch/conferenceDisplay.py?confId=2019]

\section{2-15 October 2014}

International Symposium on Crystallography-100 Years of History Fortaleza, Brazil [Info: http://www.sic100.ufc.br/ site/index.php/sic/sic100]

12-16 October 2014

Materials Science \& Technology 2014 (MS\&T 2014) David L. Lawrence Convention Center, Pittsburgh, PA, USA [Info: http://www.matscitech.org/]

\section{3-15 October 2014}

XVIII International Conference on Crystal Chemistry, X-ray Diffraction and Spectroscopy of Minerals Yekaterinburg, Russian Federation [Info: http://www.iucr. org/calendar/events/types/conferences/xviii-international-con ference-on-crystal-chemistry,-x-ray-diffraction-and-spectro scopy-of-minerals]

\section{9-22 October 2014}

GSA 2014 Vancouver, BC, Canada [Info: http://community. geosociety.org/gsa2014/home/]

26-31 October 2014

12th International Conference on X-ray Microscopy (XRM 2014) Melbourne Convention and Exhibition Centre, Melbourne, Australia [Info: http://www.xrm2014.com/]

2-5 November 2014

4th Tunisian Crystallographic Meeting (TCM4) El Mouradi Djerba Menzel, Djerba, Tunisia [Info: http://tcm4. org/]
2-6 November 2014

2014 AAPS Annual Meeting and Exposition San Diego Convention Center, San Diego, CA, USA [Info: https:// www.aaps.org/annualmeeting/]

3-7 November 2014

XIV Latin American Seminar of Analysis by X-Ray Techniques Carlos Paz, Argentina [Info: http://www. sarx2014.com.ar/index.php?title=Home]

8-15 November 2014

2014 IEEE Nuclear Science Symposium \& Medical Imaging Conference-21st Symposium on Roomtemperature Semiconductor X-ray and Gamma-ray Detectors Washington State Convention Center, Seattle, WA, USA [Info: http://nssmic2014.npss-confs.org/]

30 November-5 December 2014

2014 MRS Fall Meeting \& Exhibit Boston, MA, USA [Info: http://www.mrs.org/fall2014/]

\section{9-24 April 2015}

115th Annual Meeting of the American Roentgen Ray Society Metro Toronto Convention Centre, Toronto, Canada [Info: http://www.arrs.org/Education/Meetings/AM15/]

19-23 July 2015

2nd Asia-Oceania Conference on Neutron Scattering Sydney, Australia [Info: http://www.aocns-2015.com/]

\section{3-28 August 2015}

The 29th European Crystallographic Meeting Rovinj, Croatia [Info: http://ecm29.ecanews.org/]

13-18 September 2015

16th International Conference on Small-Angle Scattering (SAS2015) Berlin, Germany [Info: http://www.helmholtzberlin.de/events/sas/] 